\title{
A Radiation Shielding Study for the Fermilab Linac*
}

\author{
I. Rakhno, C. Johnstone \\ Fermilab, P.O. Box 500, Batavia, IL 60510
}

February 3, 2006

\begin{abstract}
Radiation shielding calculations are performed for the Fermilab Linac enclosure and gallery. The predicted dose rates around the access labyrinth at normal operation and a comparison to measured dose rates are presented. An accident scenario is considered as well.
\end{abstract}

\section{Introduction}

The Fermilab Linac presently provides an intense $400-\mathrm{MeV}$ proton beam to the Fermilab Booster for High Energy Physics and a lower energy beam to the Neutron Therapy Facility. However, the Fermilab Linac is capable of $15 \mathrm{~Hz}$ operation and many cycles remain available. A new facility, the MuCool Test Facility (MTA), is under development which takes advantage of unused Linac beam to test targets and other muon cooling apparatus which require an intense beam [1]. The full capability of the Linac, i.e. $1.3 \times 10^{13}$ protons per pulse at a $15 \mathrm{~Hz}$ repetition rate and an energy of $400 \mathrm{MeV}$, has been requested when available up to the present Linac safety envelope. (Essentially, the present envelope is about half $15 \mathrm{~Hz}$, full Linac intensity operation over one hour, so full-out operation is restricted to half-hour intervals. This envelope was based on a prior safety assessment which relied completely on distributed measurements of the radiation environment.)

Since this is a new line, it cannot be grandfathered with existing Linac shielding and Linac operation must be re-assessed. The original shielding design for the accelerator was performed almost four decades ago [2] using approximations and not very reliable, from a contemporary standpoint, data on proton and neutron interactions with matter. A reevaluation of the Linac shielding is therefore, required, especially for critical areas such as one access labyrinth located near a strong radiation source.

In this study the geometry model and beam loss model employed are described. Then, dose rate distributions in the critical area is calculated with the MARS14 code [3]. Since the labyrinth in question is instrumented, a comparison to measured dose rates is presented as well.

${ }^{*}$ The work was supported by the Universities Research Association, Inc., under contract DE-AC02$76 \mathrm{CH} 03000$ with the U. S. Department of Energy. 


\section{Geometry Model}

The geometry model developed for the area of Linac enclosure and gallery around the access labyrinth is shown if Figs. 1 and 2. As to the color scheme employed, the following convention applies: white, black, light blue, green, and grey correspond to vacuum, black hole (an artificial material used in MARS modeling that absorbs $100 \%$ of incoming radiation), air, soil, and regular concrete, respectively. The implication of other colors can vary depending on alternate materials used in the system under simulation.
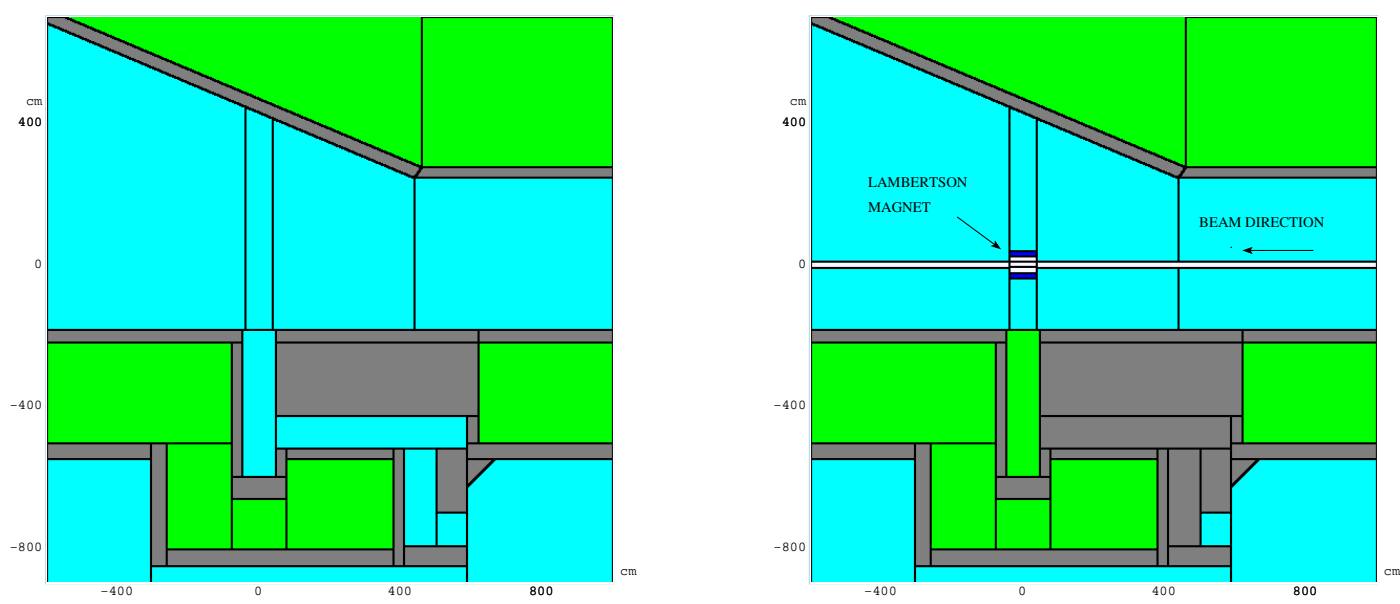

$\stackrel{\times}{\leftrightarrow \rightarrow 2}$

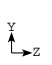

Figure 1: A plan view of the MARS geometry model of the Linac enclosure and gallery around the access labyrinth at the gallery level (left) and at the beam line level (right).
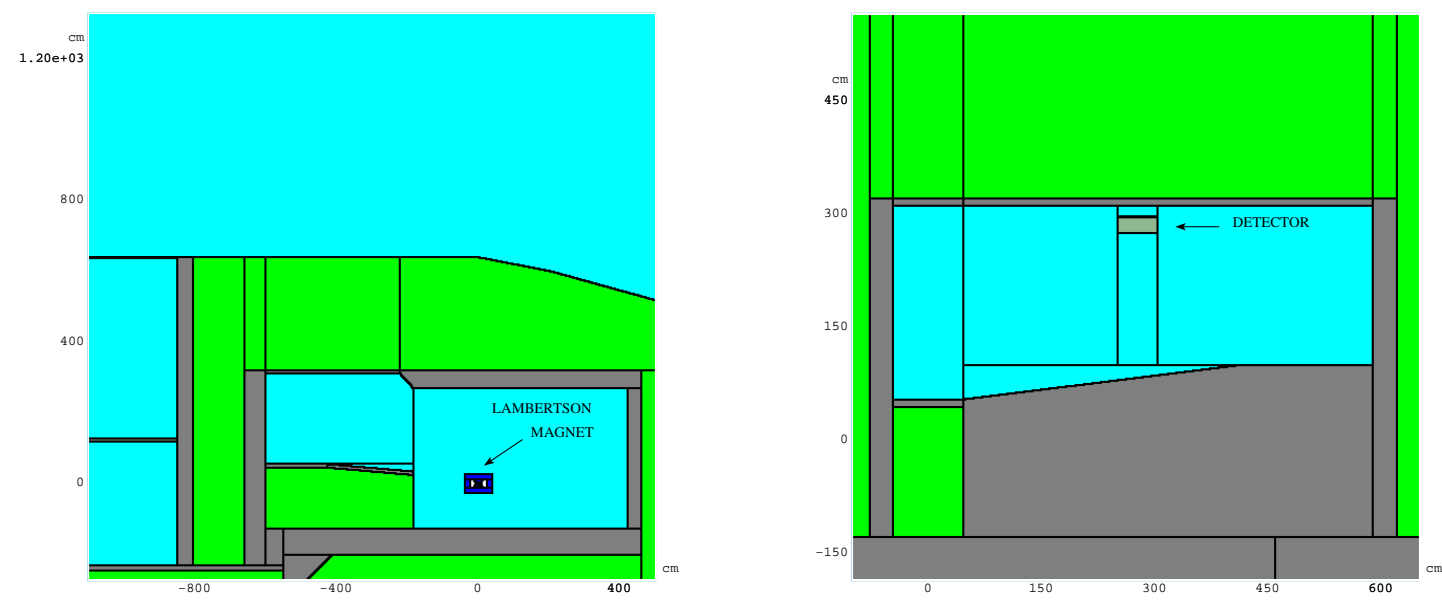

$\stackrel{x}{\rightarrow}$ y

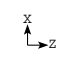

Figure 2: A cross section of the MARS geometry model of the Linac enclosure (left) and elevation view of the legs 1 and 2 in the access labyrinth (right). 
The origin of the coordinate system, $(0,0,0)$, is chosen at the geometrical center of the Lambertson magnet shown in the Figures. All the essential details that influence the radiation transport in the area are taken into account. In particular, the soil considered in the study is supposed to be compacted one with the density characteristic of the Fermilab site, i.e. 2.24 $\mathrm{g} / \mathrm{cm}^{3}$.

\section{Model of Beam Loss}

In the developed model it is assumed that the extracted primary $400-\mathrm{MeV}$ protons are lost only on the Lambertson magnet. This is a valid rationale, since the beam strikes the lambertson during Booster operation and it is here that losses are highest. For a pulse being extracted to the Booster, the beam loss occurs when the beam centroid is moved from the zero-field region (hole) in the lambertson upwards to the midplane at $X=0$ and back (see Fig. 3). The unused portion of the pulse is directed down a diagnostic line and into a beam absorber. The beam is therefore swept across the lambertson face at the beginning and end of every single extracted pulse and each beam traversal lasts $35 \mathrm{~ns}$. Since the bunchlength of a proton bunch is $5 \mathrm{~ns}$, the raw rate of the proton loss equals to

$1.6 \times 10^{9}$ protons $/$ bunch $\times 14$ lost bunches $/$ pulse $=2.24 \times 10^{10}$ protons $/$ pulse.
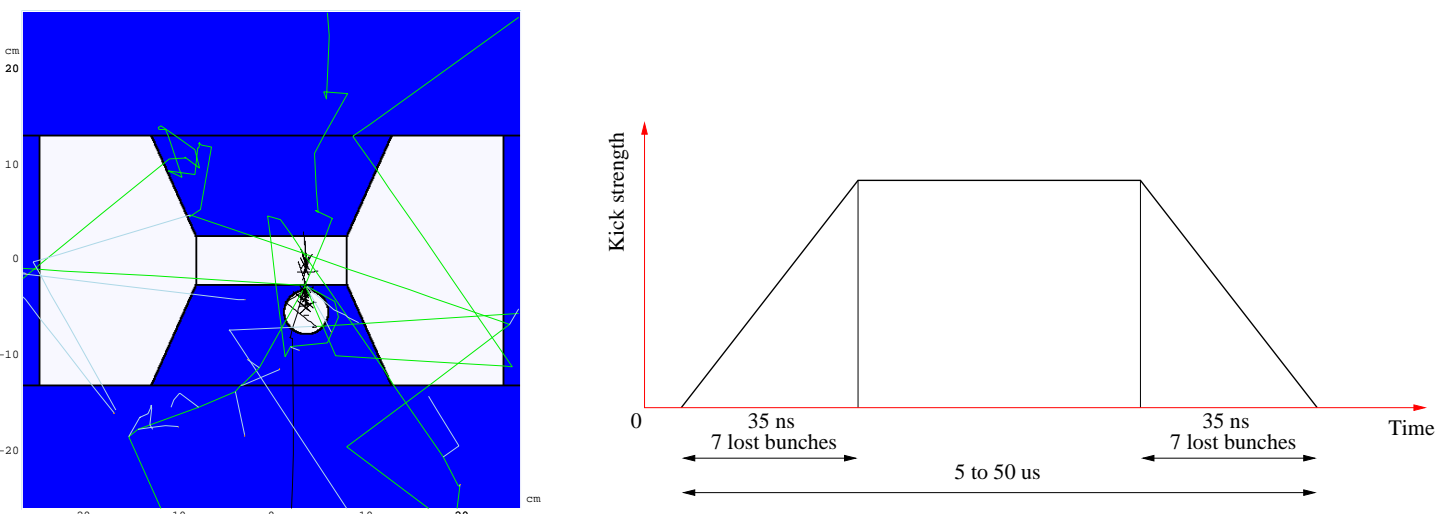

$\stackrel{x}{\leftrightarrow} \longrightarrow x$

Figure 3: The central part of the MARS model of the Lambertson magnet with sample tracks for 30 primary $400-\mathrm{MeV}$ protons and secondary particles (left) and the model describing strength of a kick $v s$ time (right). Black, green, and turquoise tracks correspond to protons, neutrons, and gammas, respectively.

The term raw loss rate is used to indicate that not all of these $2.24 \times 10^{10}$ protons are really lost on the magnet. Behavior of every single proton of the beam depends on its spatial position and direction of flight. For this reason, a realistic beam profile (see Fig. 4) is taken into account in our Monte Carlo modeling. In turn, the above-mentioned raw loss rate is used for normalization purposes. 
The proton beam parameters at $Z=38 \mathrm{~cm}$ (see Fig. 1) are given in Table 1. A cross section of the Lambertson magnet and $X Y$-projection of 30 sample proton tracks as well as tracks of secondary particles are shown in Fig. 3.

Table 1: Beam parameters at the upstream face of the Lambertson magnet.

\begin{tabular}{|c|c|c|c|}
\hline Projection & Beam type & Spatial $\sigma(\mathrm{mm})$ & Angular $\sigma(\mathrm{mrad})$ \\
\hline Horizontal & converging & 4.6 & 1.8 \\
Vertical & diverging & 7.2 & 1.75 \\
\hline
\end{tabular}
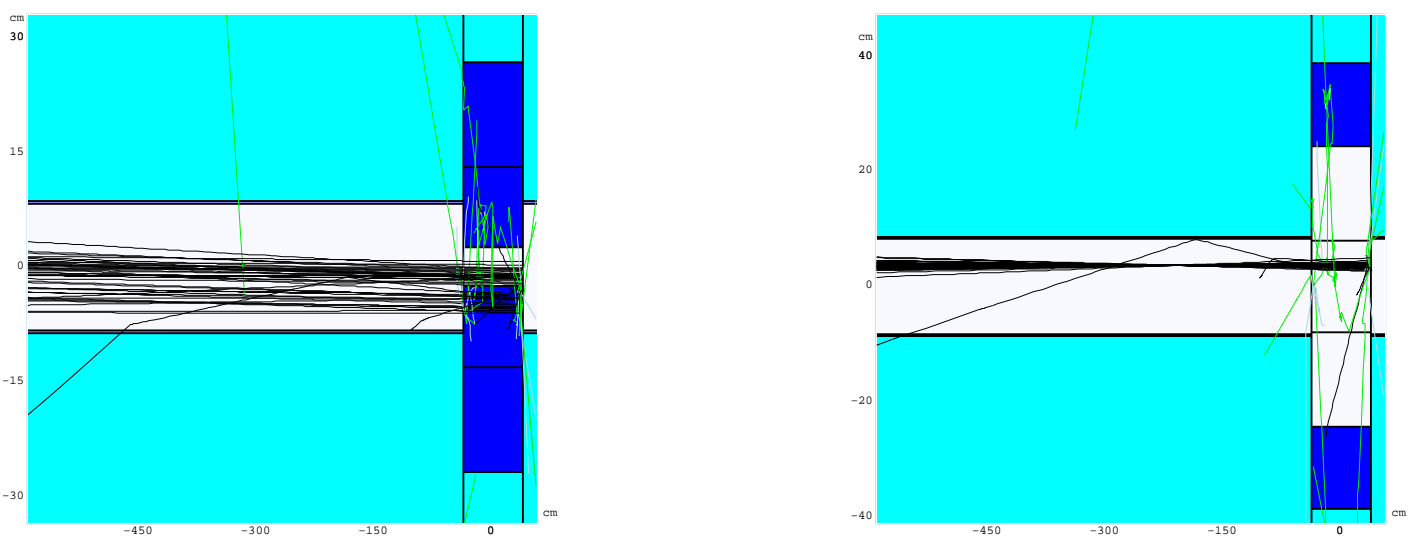

$\stackrel{x}{\iota}$

Figure 4: Vertical (left) and horizontal (right) projections of sample tracks for 30 primary protons and secondary particles shown at $Y=0$ and $X=0$, respectively. The primary beam goes through the magnet from right to left.

\section{Calculation Results}

To verify the accuracy of MARS predictions and establish a calculation-to-measurement correction factor, calculated dose rates are compared to measured ones. Afterwards the correction factor is used to predict dose rate distributions around the area.

\subsection{Comparisons to Measured Dose Rates}

In the critical labyrinth area there is a scarecrow detector, RD2040, that measures absorbed dose rate due to neutrons and gammas. It is mounted on the wall in the leg 2 of the labyrinth (see Figs. 1 and 2). The detector performs continuous on-line monitoring in the area. The measured dose rate is not constant but reveals significant, irregular oscillations around the average level of $171 \mathrm{mrem} / \mathrm{hr}$ as shown in Fig. 5. Since the repetition rate of the Linac varies, this is expected. The scarecrow detectors provide a high sensitivity ( $25 \mu \mathrm{rem} / \mathrm{count}$ ) 
to measure relatively high dose rates - above a few mrem/hr. Therefore, in the following we compare calculated dose rates to measured data taken in a high radiation area. For outer shielding regions, where dose rate should approach the natural background, more sensitive detectors are usually used (e.g. chipmunks that provide $2.5 \mu \mathrm{rem} /$ count). Due to a limited scope of this study, we do not perform comparisons between measurements and calculations for the outer regions.

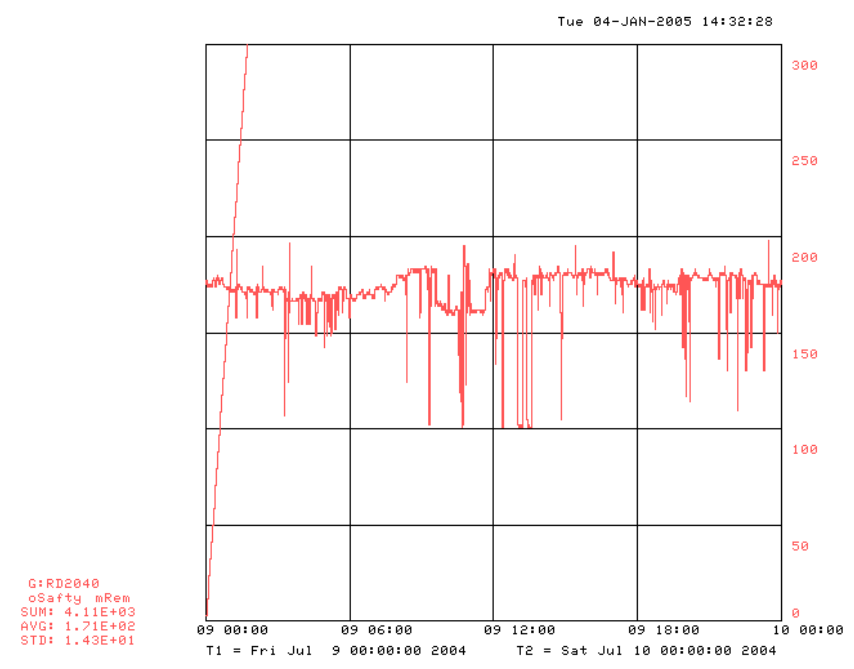

Figure 5: The dose rate (mrem/hr) measured with the scarecrow detector, RD2040, placed in the leg 2 of the access labyrinth [4]. The readings were taken for 24 hours from midnight on July 9, 2004 till midnight on July 10, 2004. The background dose rate of the detector due to a built-in radiation source is equal to $100 \mathrm{mrem} / \mathrm{hr}$. The line going upward represents the integral measured dose $v s$ time.

A proper normalization of the calculated dose rate requires also some data on amount of delivered protons. Specifically, the number of delivered beam pulses is required in order to compare with the beam loss model. The total number of protons delivered in a specfic time interval is therefore used rather than the repetition rate. The data on delivered beam pulses is stored in a database back to June 2000 [5]. For this comparison we have chosen, arbitrarily, three different time intervals with different durations:

- July 9-10, 2004 (24 hours);

- July 1-8, 2004 (168 hours);

- July 1-21, 2004 (480 hours).

The data on the measured dose rates for the second and third intervals are shown in Fig. 6. The corresponding data on beam pulses delivered in July 2004 is given in Table 2.

For the comparison we use the integral measured doses shown in Figs. 5 and $6(4.11 \times$ $10^{3}, 2.83 \times 10^{4}$, and $\left.8.34 \times 10^{4} \mathrm{mrem}\right)$. The integral doses are divided by the corresponding number of beam pulses and the background dose rate in the detector $(100 \mathrm{mrem} / \mathrm{hr}) \mathrm{has}$ 

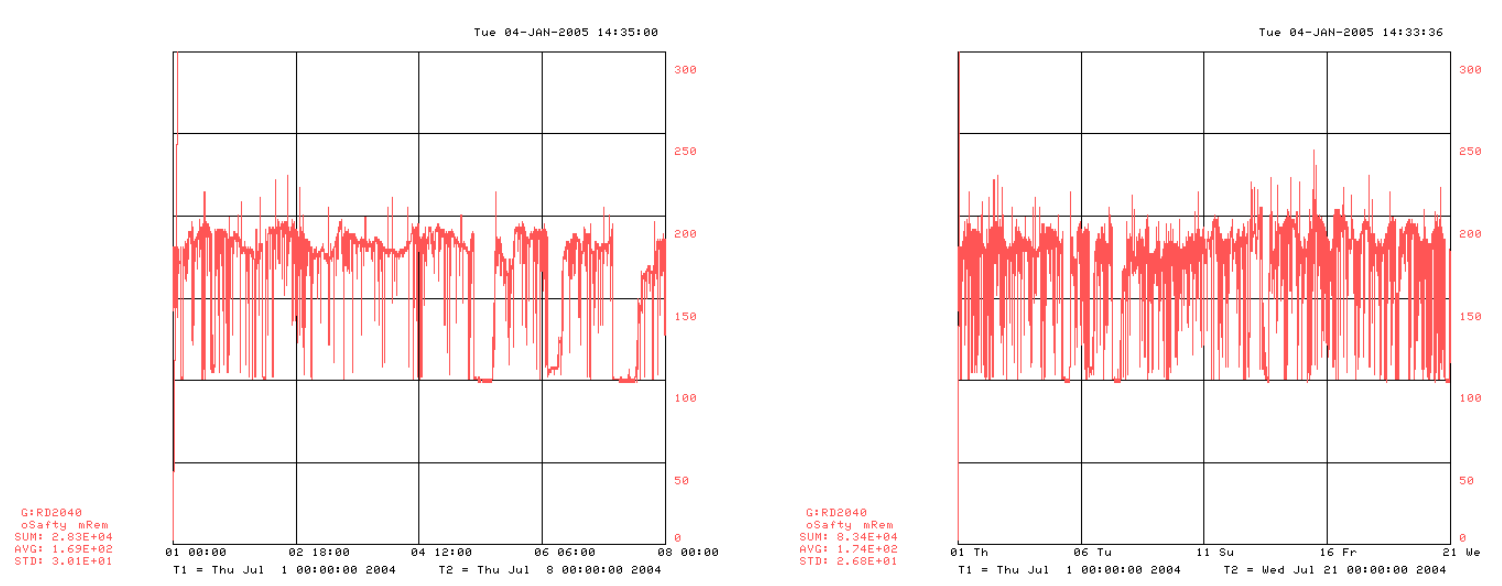

Figure 6: The same as in Fig. 5 but for July 1-8, 2004 (left) and July 1-21, 2004 (right) [4]. Average measured dose rate for the time intervals equals to 169 and $174 \mathrm{mrem} / \mathrm{hr}$, respectively.

Table 2: Number of beam pulses delivered daily by Linac in July 2004 [5]. Each counting procedure was completed at 1 a.m. on the shown date.

\begin{tabular}{|l|c|l|c|l|c|}
\hline Date & Number of pulses & Date & Number of pulses & Date & Number of pulses \\
\hline June 30 & 340720 & July 8 & 215816 & July 16 & 365335 \\
July 1 & 354023 & July 9 & 319186 & July 17 & 378149 \\
July 2 & 348599 & July 10 & 308453 & July 18 & 396287 \\
July 3 & 351403 & July 11 & 348656 & July 19 & 326482 \\
July 4 & 365828 & July 12 & 354645 & July 20 & 370878 \\
July 5 & 382356 & July 13 & 328635 & July 21 & 269648 \\
July 6 & 259353 & July 14 & 304930 & July 22 & 324739 \\
July 7 & 302493 & July 15 & 371634 & & \\
\hline
\end{tabular}

been eliminated as well. Results of the comparison are presented in Table 3. One can see from the Table that the calculation overestimates the dose rate. This is not unexpected given the number of components and obstacles near the Lambertson, and the disagreement is not significant from a practical shielding standpoint. Further, when considering the MARS predictions for the dose distributions around the area, the calculated dose rates can be multiplied by a correction factor of $1 /(1+0.35)$. A possible dependence of the correction factor on particle spectra, i.e. on spatial location is ignored.

It should be noted also that, having eliminated the repetition rate and considering only dose per pulse, we should not observe any meaningful difference between the data taken during the three time intervals. In other words, all the beam pulses are the same from the standpoint of beam loss on the Lambertson magnet. The first and third data, $5.53 \times 10^{-3}$ and $5.54 \times 10^{-3} \mathrm{mrem} /$ pulse, confirm the statement. The second time interval, however, breaks the rule. The observed difference indicates that additional beam loss modes came into play from July 1 till July 8, 2004. This can likely be explained from a tuning standpoint 
and serves to estimate limits on different operational parameters of the Linac.

Table 3: A comparison between measured and calculated dose rates (mrem/pulse) in the leg 2 of the access labyrinth. The fraction of the beam deducted due to operations of the Neutron Therapy Facility (NTF) is shown as well.

\begin{tabular}{|l|c|c|c|}
\hline Time interval & $\begin{array}{c}\text { Dose rate measured with } \\
\text { scarecrow detector RD2040 } \\
\text { (background 100 mrem/hr) }\end{array}$ & $\begin{array}{c}\text { Dose rate calculated } \\
\text { with MARS code } \\
(1 \sigma=15 \%)\end{array}$ & $\frac{C-E}{E}$ \\
\hline $\begin{array}{l}\text { July 9-10, 2004 } \\
\text { NTF } \rightarrow 0 \%\end{array}$ & $\frac{4.11 \times 10^{3} \text { mrem }}{3.085 \times 10^{5} \text { pulses }} \times \frac{71}{171}=5.53 \times 10^{-3}$ & $7.48 \times 10^{-3}$ & $35 \%$ \\
$\begin{array}{l}\text { July 1-8, 2004 } \\
\text { NTF } \rightarrow 0.2 \%\end{array}$ & $\frac{2.83 \times 10^{4} \text { mrem }}{2.23 \times 10^{6} \text { pulses }} \times \frac{69}{169}=5.19 \times 10^{-3}$ & $7.48 \times 10^{-3}$ & $44 \%$ \\
$\begin{array}{l}\text { July 1-21, 2004 } \\
\text { NTF } \rightarrow 4 \%\end{array}$ & $\frac{8.34 \times 10^{4} \text { mrem }}{6.67 \times 10^{6} \text { pulses } \times 0.96} \times \frac{74}{174}=5.54 \times 10^{-3}$ & $7.48 \times 10^{-3}$ & $35 \%$ \\
\hline
\end{tabular}

Finally, possible reasons for disagreement between calculation and measurement are listed below:

- Non-Lambertson beam loss is not considered. It should increase the calculated dose rate.

- Real material density of the concrete may differ from the value used in these calculations $\left(2.35 \mathrm{~g} / \mathrm{cm}^{3}\right)$. The same applies to concrete composition. This correction could either increase or decrease the calculated dose rate.

- Non-uniform sampling of the beam centroid on the way between the midplane and the field-free region could be more realistic. This correction also could either increase or decrease the calculated dose rate.

- Magnetic field inside the Lambertson magnet was ignored. It is an acceptable approximation as far as the neutral secondaries, neutrons and gammas, make the dominating contribution to the prompt dose outside the shielding.

All that matters as far as the disagreement between the calculation and measurement within a few tens of percent is concerned. 


\subsection{Calculated Dose Rate Distributions}

The distributions of prompt dose rate, calculated for normal operation at the repetition rates of 4 and $15 \mathrm{~Hz}$, are presented in Figs. 7 and 8.

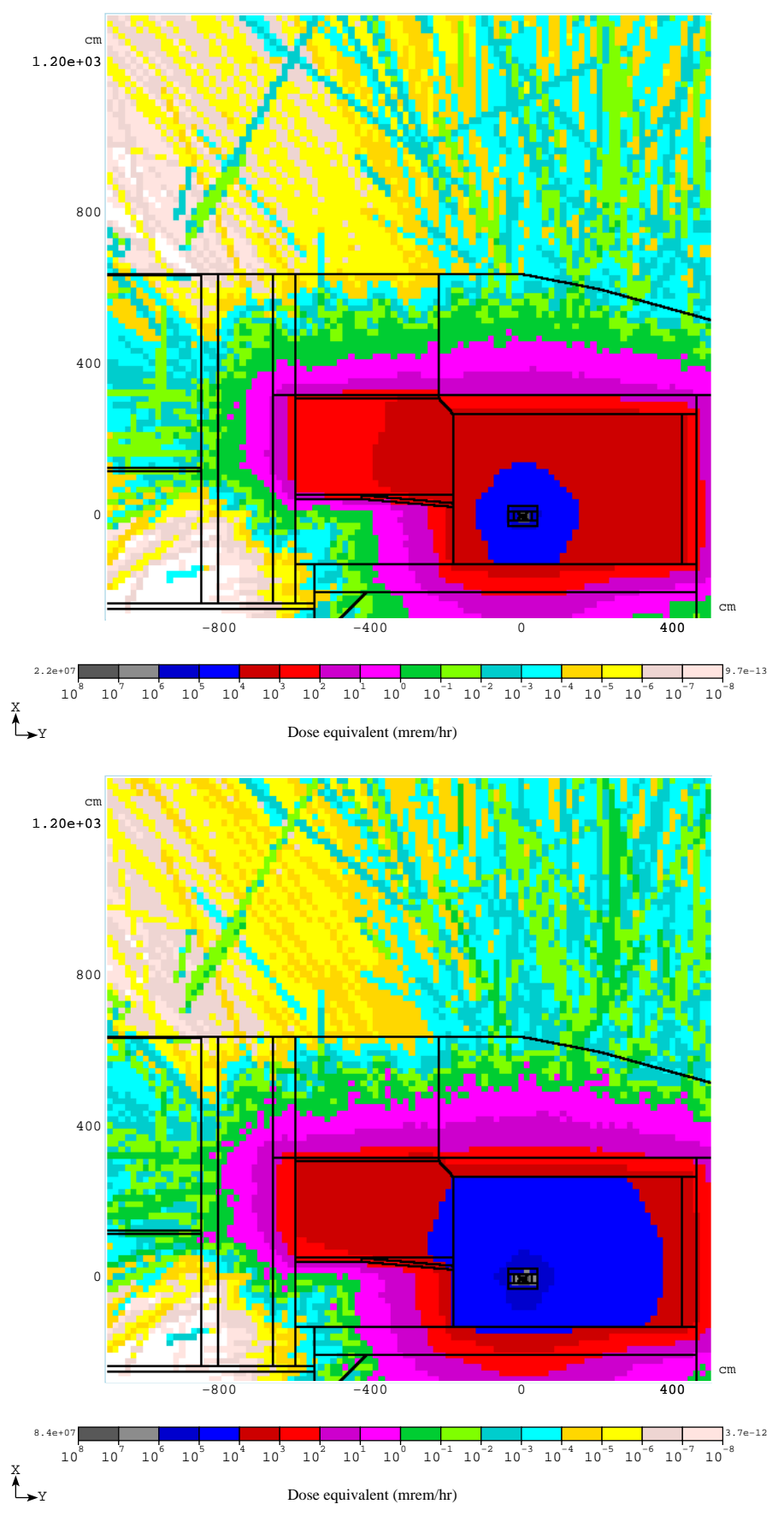

Figure 7: Dose rate distributions in the cross section of the Linac gallery and enclosure calculated at the repetition rate of 4 (top) and $15 \mathrm{~Hz}$ (bottom) for the vertical slice as thick as $100 \mathrm{~cm}$, namely $-50 \mathrm{~cm} \leq Z \leq 50 \mathrm{~cm}$. 

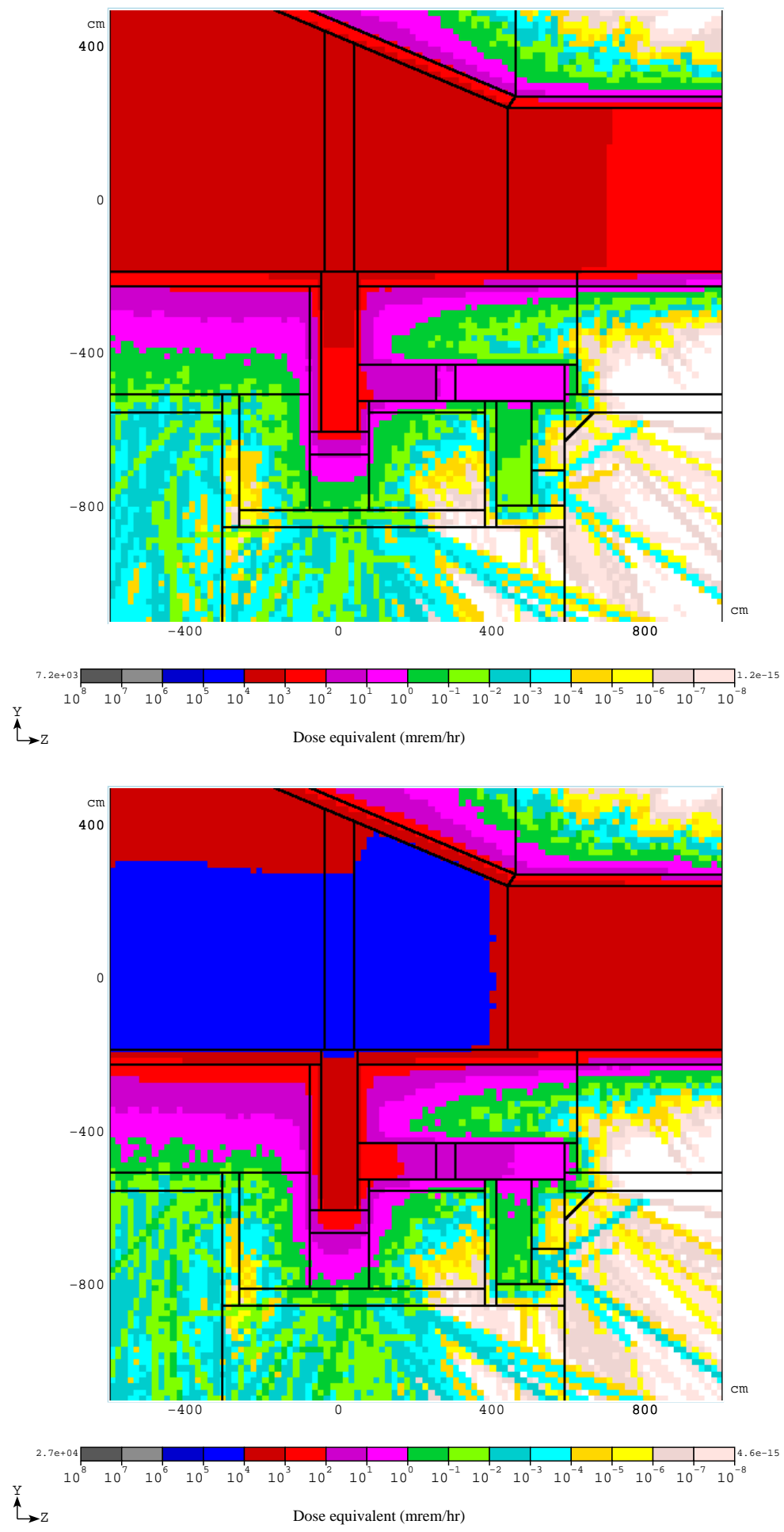

Figure 8: Dose rate distributions (plan view) for the Linac gallery and enclosure calculated at the repetition rate of 4 (top) and $15 \mathrm{~Hz}$ (bottom) for the horizontal slice as thick as 170 $\mathrm{cm}$, namely $130 \mathrm{~cm} \leq X \leq 300 \mathrm{~cm}$. 


\subsubsection{Linac Enclosure and Gallery}

One can see from Fig. 7 that the prompt dose rate at the lower level of the Linac gallery $(X<120 \mathrm{~cm}$ and $Y<-850 \mathrm{~cm}$ in Fig. 2) in the area accessible to people is below the level of $0.05 \mathrm{mrem} / \mathrm{hr}$ even for the repetition rate of $15 \mathrm{~Hz}$. The small penetrations along the wall connecting the personnel gallery and the enclosure were not considered, but their contribution should be much less and not change the radiation grade.

The thickness of the layer used to calculate the horizontal dose distribution in Fig. 8 was chosen to represent an average human, and thus the dose distribution is applicable to a person in the Linac gallery on the upper level. One can see that at the repetition rate of 4 $\mathrm{Hz}$ the dose rate in the gallery is below $0.05 \mathrm{mrem} / \mathrm{hr}$ which allows it to be qualified as an area of unlimited occupancy according to Ref. [6]. At $15 \mathrm{~Hz}$, however, this is not the case. In the area around $Z=0$ and $Y \leq-850 \mathrm{~cm}$ (see Fig. 8) one obtains, using the exponential attenuation law and correction factor described above, the following dose

$$
10 \mathrm{mrem} / \mathrm{hr} \times \exp (-135 / 38.7) /(1+0.35)=0.22 \mathrm{mrem} / \mathrm{hr},
$$

where 38.7 is the dose attenuation length (in $\mathrm{cm}$ ) in the compacted soil [7]. It greater than the required level $(0.05 \mathrm{mrem} / \mathrm{hr})$ by a factor of 4.5 . To provide an additional shielding, a removable block could be used in leg 1 of the labyrinth (see Fig. 9) to reduce the dose back to required limits. However, this shield block may require removal for even short accesses, so it's operational impact requires evaluation.
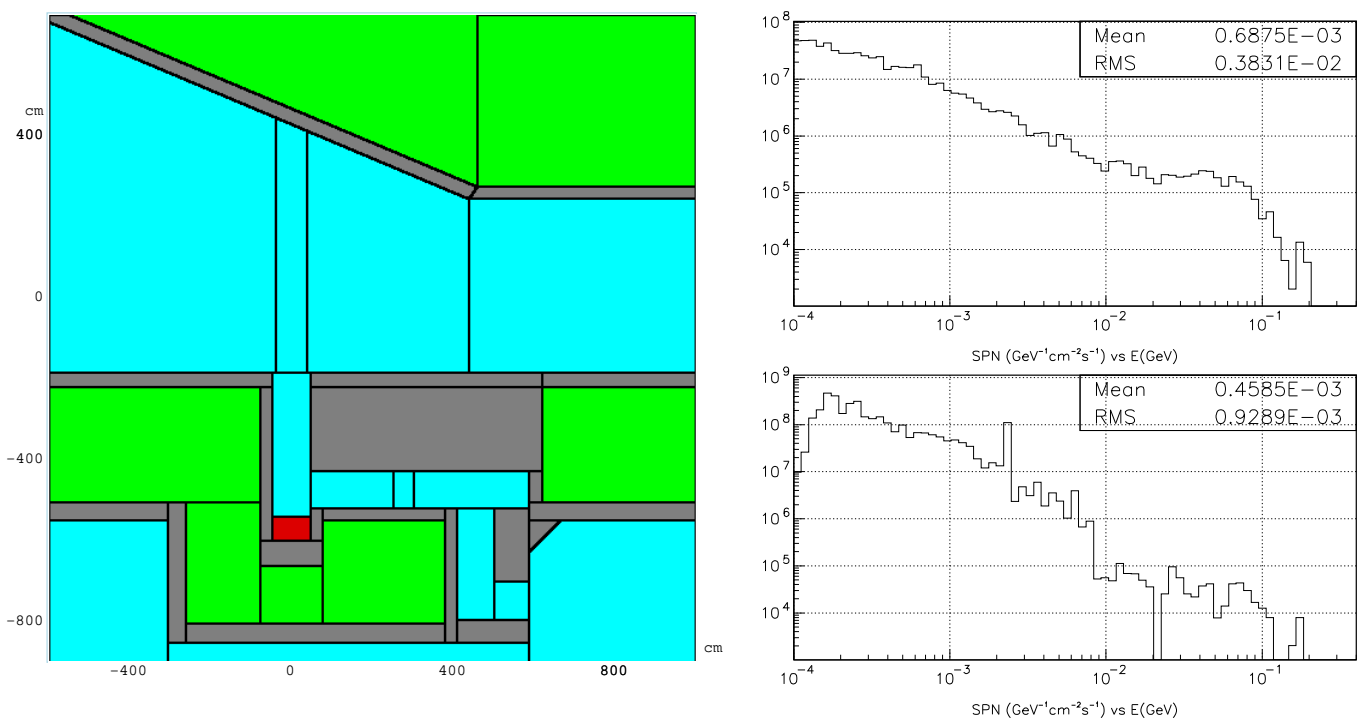

$\stackrel{0}{\leftrightarrow \rightarrow 2}$

Figure 9: A plan view of the MARS geometry model of the access labyrinth with a removable shielding block $(55 \mathrm{~cm} \leq X \leq 310 \mathrm{~cm},-600 \mathrm{~cm} \leq Y \leq-540 \mathrm{~cm},-50 \mathrm{~cm} \leq Z \leq 50 \mathrm{~cm})$ (left) and the calculated neutron and gamma spectra over the corresponding air volume in the leg 1 (right top and right bottom, respectively). 
To choose an appropriate material, particle spectra have been calculated over the corresponding volume in the leg 1 which serves, in fact, as a neutron trap. The spectra shown in Fig. 9 indicate that the area is populated mostly by low-energy neutrons and gammas while the contribution from charged hadrons can be neglected. Therefore, polyethylene can be used as a candidate material for the removable block. It has been determined that the block with dimensions shown in Fig. 9 provides an additional ten-fold dose reduction at upper level of the gallery. Thus, with the block in place, the hottest spot in the gallery near the end of leg 1 will be about $0.02 \mathrm{mrem} / \mathrm{hr}$. One can reduce thickness of the block in $Y$-direction from 60 to $40 \mathrm{~cm}$ to have $0.05 \mathrm{mrem} / \mathrm{hr}$ at this spot. The calculations performed revealed also that the block gives rise to a slight increase of dose rate in leg 2 due to backscattering. The increase, however, can be neglected because the dose rate in the gallery near the end of leg 2 (see Fig. $8, Y=-600 \mathrm{~cm}$ and $Z=600 \mathrm{~cm}$ ) is below $0.05 \mathrm{mrem} / \mathrm{hr}$.

According to our estimates, at upper level of the gallery in the area $Z \leq-300 \mathrm{~cm}$ and $Y \leq-540 \mathrm{~cm}$ (see Fig. 8) the dose rate at the repetition rate of $15 \mathrm{~Hz}$ with this shield block in place will be about $0.05 \mathrm{mrem} / \mathrm{hr}$.

\subsubsection{Berm}

From the calculated distributions (see Fig. 7) one can estimate that, at the repetition rate of $4 \mathrm{~Hz}$, the dose rate on the berm is below $0.05 \mathrm{mrem} / \mathrm{hr}$, so that it can be qualified as area of unlimited occupancy. At $15 \mathrm{~Hz}$, starting at the dose rate level of $1 \mathrm{mrem} / \mathrm{hr}$ and using the attenuation length and correction factor described above, one obtains the following dose rate on the berm

$$
1 \mathrm{mrem} / \mathrm{hr} \times \exp (-82 / 38.7) /(1+0.35)=0.09 \mathrm{mrem} / \mathrm{hr} .
$$

To qualify the berm as an area of unlimited occupancy at $15 \mathrm{~Hz}$, the additional two-fold dose reduction can be provided with appropriate local shielding inside the enclosure.

\subsubsection{An Accident Scenario}

According to previous beam optics calculations for the Linac beam line, it is assumed in an accident scenario that the beam experiences an upward kick at the Q2 quadrupole and hits the beam pipe downstream. Trajectories of 50 sample lost protons are shown in Fig. 10. A numerical description of the accident is the following:

- Kick by Q2 as large as 50 mrad.

- The misbehaved beam hits the beam pipe $1 \mathrm{~m}$ upstream the Lambertson magnet.

- Gaussian beam profile with $\sigma_{h}$ and $\sigma_{v}$ equal to 9 and $3 \mathrm{~mm}$, respectively $(\sigma$ values for corresponding angles equal to 0.45 and $1.4 \mathrm{mrad}$, respectively).

One also assumes that full Linac intensity (at the repetition rate of $15 \mathrm{~Hz}$ ) can be lost in such an accident and its duration is equal to 1 second. This is a very conservative estimate for the response time of the Linac control system. A more realistic estimate can be derived 
from the consideration of beam loss monitors (BLM) employed in the system. The BLMs were fashioned after the models that are used in the Tevatron $[5,8]$. The basic operational features of the monitors are as follows. The current from the BLM ionization chamber goes directly into the log integrator with an RC time constant. Then, through an amplifier, the current goes as the input into the alarm/abort comparators. It is the alarm/abort circuit that finally decides whether to generate a hardware alarm/abort signal. The RC time constant for the beam loss monitors used in the Linac control system equals to $344 \mu \mathrm{sec}$ and the system samples the monitor signals at a time specified by a timer setting [9]. If the system detects a mis-behaved pulse, the beam will be turned off and there will be no further beam loss. In other words, during an accident only one beam pulse can be lost. Therefore, when considering an accident that lasts one second, one incorporates a safety factor of about 10 .

The calculated distribution of the total dose in such an accident is shown in Fig. 10. One can determine from the Figure, using the same attenuation and correction procedure as in previous sections, that the integral dose on the berm due to the accident is equal to 0.3 mrem. According to Ref. [6], no precautions are needed if the maximum dose expected in 1 hour is less than 1 mrem.

One considers only berm because it is beam that is mis-steered upward that creates the severest potential exposure. To estimate the dose for a hypothetical horizontal kick of the same strength directed toward the access labyrinth, we can use the obtained scaling factor "integral dose at accident/prompt dose," namely " $0.3 \mathrm{mrem} / 0.09 \mathrm{mrem} / \mathrm{hr}$ ". It follows from the scaling and dose distributions presented above for the access labyrinth that the accident with the horizontal beam kick also complies with the " 1 mrem in 1 hour" requirement of Ref. [6].

\section{Conclusions}

The study was performed to resolve the radiation shielding issues for the Linac taking into account the planned new beamline installation. Calculations were performed for the maximum beam intensity and repetition rate $(15 \mathrm{~Hz})$. The Linac enclosure and gallery around the access labyrinth were investigated as they represent areas of human occupancy and potential exposure.

A comparison to the dose rates measured with a scarecrow detector was performed. It was shown that the MARS predictions overestimate the dose rates by 35\%. The corresponding correction factor was used to renormalize results of further studies.

Within the framework of a credible accident scenario, a beam accident at the Linac enclosure complies with the "1 mrem in 1 hour" requirement of Ref. [6], so that no precautions are needed.

At normal operation at the repetition rate of $4 \mathrm{~Hz}$, all areas investigated qualify as areas of unlimited occupancy where the dose rate does not exceed $0.05 \mathrm{mrem} / \mathrm{hr}$ [6]. At $15 \mathrm{~Hz}$, however, additional shielding is required in leg 1 of the access labyrinth and in the Linac enclosure (e.g. a removable block and/or local shielding around the Lambertson magnet, respectively) in order that these areas again qualify as areas of unlimited occupancy. 

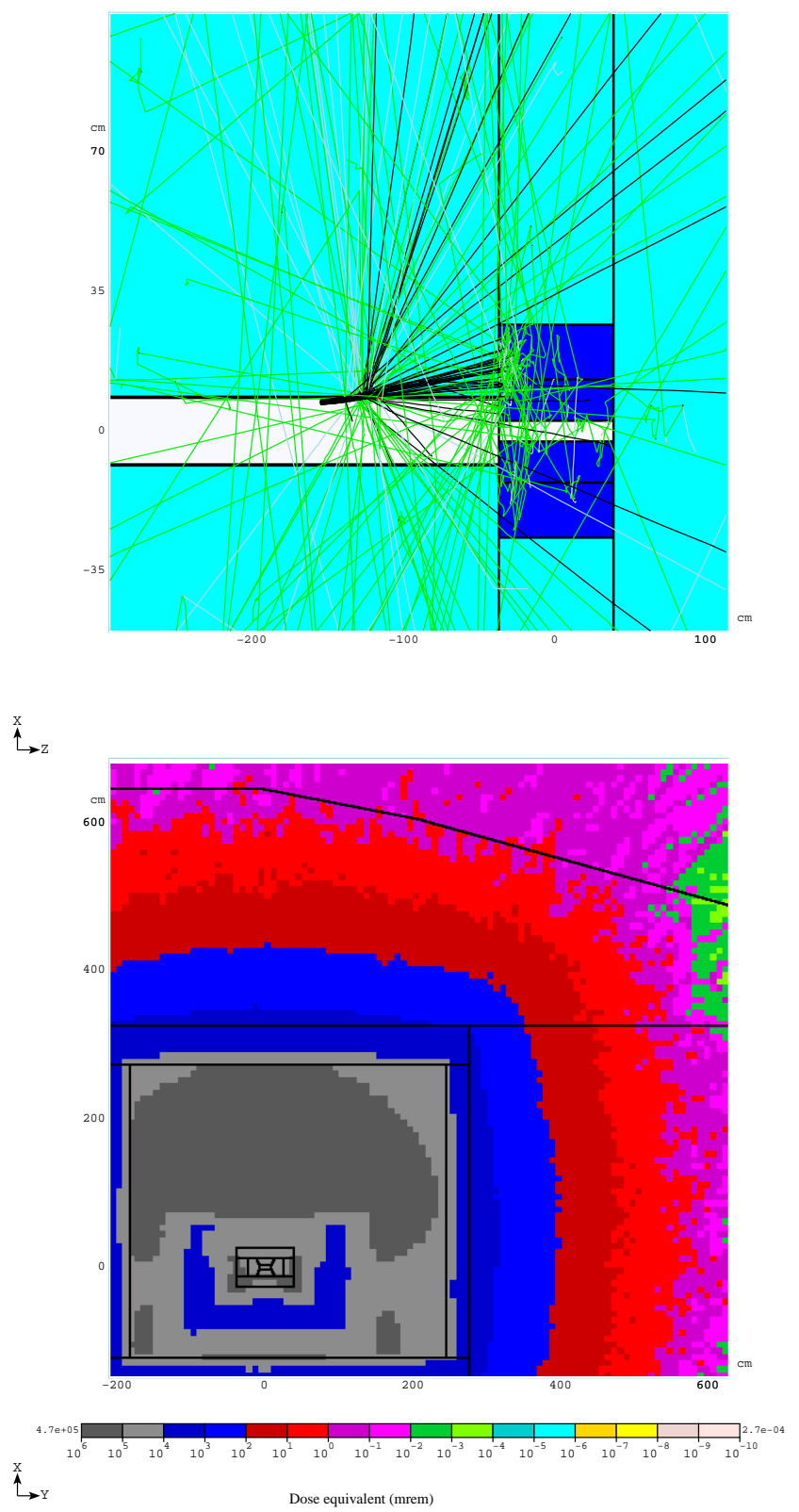

Figure 10: A vertical projection of sample tracks for 50 lost primary protons and all the secondaries shown at $Y=0$ (top) and integral dose distribution over the Linac enclosure (bottom) calculated for the described accident scenario. Black, green, and turquoise tracks correspond to protons, neutrons, and gammas, respectively.

\section{Acknowledgements}

The authors are thankful to Craig Moore, Michael Gerardi, Gary Lauten, William Higgins, John Larson, Elliott McCrory, Larry Allen, and Nikolai Mokhov of Fermilab for helpful discussions. 


\section{References}

[1] http://www.fnal.gov/projects/muon_collider/cool/cool.html; D. Errede, R. Alber, A. Bross et al. Proc. of the 2003 Part. Accel. Conf., Portland, OR, USA, May 2003.

[2] M. Awschalom, “Linac Shielding,” Fermilab Note FN-129, April 1968.

[3] N.V. Mokhov, "The MARS Code System User's Guide," Fermilab-FN-628 (1995);

N. V. Mokhov, O. E. Krivosheev, "MARS Code Status," Proc. of the Monte Carlo 2000 Conference, Lisbon, October 23-26, 2000, Springer, p. 943; Fermilab-Conf-00/181 (2000); http://www-ap.fnal.gov/MARS/

[4] G. Lauten, Private communication, Fermilab, January 2005.

[5] E. McCrory, Private communication, Fermilab, January 2005.

[6] "Fermilab Radiological Control Manual," Article 236, http://www-esh.fnal.gov/FRCM/.

[7] I. Rakhno, C. Johnstone, "Radiation Shielding Calculations for MuCool Test Area at Fermilab," Fermilab-TM-2248 (2004).

[8] J. Larson, F. Krueger, "Beam Loss Monitor System Description," Fermilab, TM $158499,1983$.

[9] L. Allen, Private communication, Fermilab, September 2005. 